\title{
Effects of popular diets on anthropometric and metabolic parameters: an umbrella review of meta-analyses of randomized controlled trials
}

\author{
Monica Dinu ${ }^{1}$, Giuditta Pagliai $^{1}$, Donato Angelino ${ }^{2}$, Alice Rosi ${ }^{3}$, Margherita Dall'Asta ${ }^{3}$, \\ Letizia Bresciani ${ }^{2}$, Cinzia Ferraris ${ }^{4}$, Monica Guglielmetti ${ }^{4}$, Justyna Godos ${ }^{5}$, Cristian Del Bò ${ }^{6}$, \\ Daniele Nucci ${ }^{7}$, Erika Meroni ${ }^{6}$, Linda Landini ${ }^{8}$, Daniela Martini ${ }^{2}$ and Francesco Sofi ${ }^{1}$ \\ ${ }^{1}$ Department of Experimental and Clinical Medicine, University of Florence, Florence, Italy, \\ ${ }^{2}$ Human Nutrition Unit, Department of Veterinary Science, University of Parma, Parma, Italy, \\ ${ }^{3}$ Human Nutrition Unit, Department of Food and Drug, University of Parma, Parma, Italy, \\ ${ }^{4}$ Human Nutrition and Eating Disorder Research Center, Department of Public Health, Experimental and Forensic \\ Medicine, University of Pavia, Pavia, Italy, \\ ${ }^{5}$ Department of Biomedical and Biotechnological Sciences, University of Catania, Catania, Italy, \\ ${ }^{6}$ Department of Food, Environmental and Nutritional Sciences (DeFENS), University of Milan, Milan, Italy, \\ ${ }^{7}$ Digestive Endoscopy Unit, Veneto Institute of Oncology IOV-IRCCS, Padua, Italy and \\ ${ }^{8}$ Medical Affairs Sanofi-Genzyme, Milan, Italy
}

\begin{abstract}
Introduction: The prevalence of overweight, obesity, and their related complications is increasing worldwide. We aimed to summarise and critically evaluate the effects of different popular diets on anthropometric parameters and metabolic risk factors.

Material and methods: An umbrella review of meta-analyses of randomized controlled trials was conducted according to the Joanna Briggs Institute Umbrella Review Methodology. The review protocol has been registered on PROSPERO (ID: CRD42019126103). Medline, Embase, Scopus, Cochrane Database of Systematic Reviews, and Web of Science were searched from inception to April 2019 to identify meta-analyses of randomized controlled trials that examined the effects of different diets on anthropometric parameters and metabolic risk factors. For each association, we estimated the summary effect size by random-effects and fixed-effects models, the $95 \%$ confidence interval, and the $95 \%$ prediction interval. We also assessed the between-study heterogeneity and evidence for small-study effects. We further applied standardized methodological criteria to evaluate the epidemiological credibility of the statistically significant associations.
\end{abstract}

Results: Overall, 80 articles reporting 495 unique meta-analyses were examined, covering a wide range of popular diets: lowcarbohydrate $(n=21$ articles), high-protein $(n=8)$, low-fat $(n=9)$, palaeolithic $(n=2)$, low glycaemic index/load $(n=12)$, intermittent energy restriction $(n=6)$, Mediterranean $(n=11)$, Nordic $(n=2)$, vegetarian $(n=9)$, Dietary Approaches to Stop Hypertension or DASH $(n=6)$, and portfolio dietary pattern $(n=1)$. The methodological quality of most articles $(n=65 ; 81 \%)$, evaluated using the AMSTAR-2 questionnaire, was low or critically low. The strength of evidence was generally weak. The most consistent evidence was reported for Mediterranean diet, with suggestive evidence of an improvement in weight, body mass index (BMI), total cholesterol, glucose and blood pressure. Suggestive evidence of an improvement in weight and blood pressure was also reported for DASH diet. Low-carbohydrate, high-protein, low-fat and low-glycaemic index/load diets showed suggestive and/or weak evidence of a reduction in weight and BMI, but contrasting evidence for lipid, glycaemic and blood pressure parameters, suggesting potential risks of unfavourable effects. Finally, evidence for palaeolithic, intermittent energy restriction, Nordic, vegetarian and portfolio dietary patterns was graded as weak or not statistically significant.

Discussion: Most meta-analyses showed low methodological quality and the strength of evidence, assessed using evidence classification criteria, was generally weak. Among all the diets evaluated, Mediterranean diet had the strongest and most consistent evidence of a positive effect on both anthropometric parameters and metabolic risk factors.

\section{Conflict of Interest}

There is no conflict of interest 